\title{
CARACTERIZANDO O ENSINO DO PENSAMENTO COMPUTACIONAL NA EDUCAÇÃO INFANTIL E NO ENSINO FUNDAMENTAL (ANOS INICIAIS) NO BRASIL
}

\section{CHARACTERIZING THE TEACHING OF COMPUTATIONAL THINKING IN KINDERGARTEN AND IN THE EARLY YEARS OF ELEMENTARY EDUCATION IN BRAZIL}

\begin{abstract}
Tanci Simões Gomes, Universidade Federal de Pernambuco I CESAR School, tancicleide.gomes@gmail.com, https://orcid.org/0000-0002-7151-9585
\end{abstract}

Taciana Pontual Falcão, Universidade Federal Rural de Pernambuco, taciana.pontual@ufrpe.br, http://orcid.org/0000-0003-2775-4913

Patricia Tedesco, Universidade Federal de Pernambuco, pcart@ cin.ufpe.br, https://orcid.org/0000-0001-9450-9219

\begin{abstract}
Resumo. O Pensamento Computacional (PC) abrange habilidades relevantes para toda a sociedade. Pesquisas recentes sugerem que todos deveriam ter a oportunidade de desenvolvê-lo, o que implica em sua integração desde a Educação Infantil. No entanto, as crianças em fase de letramento possuem necessidades que exigem abordagens, métodos e ferramentas específicos. No intuito de delinear o contexto nacional a partir deste prisma, foi realizado um mapeamento sistemático da literatura abrangendo os estudos publicados entre 2008 e 2021, para caracterizar a experiência de ensino do PC na Educação Infantil e nos anos iniciais do Ensino Fundamental. Os resultados obtidos apontam que existem poucos estudos voltados à Educação Infantil e que, atualmente, as principais iniciativas para o desenvolvimento do PC para crianças pequenas focam no uso de atividades desplugadas.
\end{abstract}

Palavras-chave: educação em computação, pensamento computacional, educação infantil, ensino fundamental.

Abstract. Computational Thinking (CT) encompasses skills that are relevant to society. Recent research suggests that everybody should be able to develop it, which entails its integration in Early Childhood and Elementary Education. However, children in the literacy stage have needs that demand specific approaches, methods and tools. With the aim of tracing a profile of the national context, we have carried out a systematic literature mapping encompassing studies published from 2008 to 2021, to characterize the experience of teaching CT in Early Childhood and in the early years of Elementary School. The results obtained indicate that there are few studies focusing on early childhood education as well as that current initiatives for developing CT in young children focus on using unplugged activities.

Keywords: computing education, computational thinking, kindergarten, elementary school.

\section{INTRODUÇÃO}

Em uma sociedade amplamente conectada, com as tecnologias tornando-se cada vez mais pervasivas, o desenvolvimento de habilidades como alfabetização e letramento digital têm sido amplamente discutidos. No entanto, muitos estudos têm argumentado que apenas estas habilidades não são suficientes, e apontam que o desenvolvimento do pensamento computacional (PC) é uma necessidade elementar para todos os cidadãos. 
Para além das diferentes compreensões, mais ou menos abrangentes, o PC tem sido considerado tão relevante quanto a leitura e a escrita (Wing 2006, Wing 2008), de sorte que qualquer cidadão deve ter a chance de aprender seus fundamentos tão cedo e de maneira tão acessível quanto Linguagem ou Matemática (Guzdial 2015, Wing 2008).

Não existe um consenso sobre a definição do PC. Alguns autores apontam que o PC se baseia em fundamentos da Ciência da Computação e envolve um conjunto de habilidades como resolução de problemas, programação e pensamento analítico (IFTF 2009, Stephenson et al. 2012). Outros argumentam que o PC compreende o aumento do poder cognitivo, a criatividade e novas maneiras de descobrir o mundo (Barbra 2016, Blikstein 2008). Se considerarmos o PC a partir de uma perspectiva mais ampla, podemos afirmar que as discussões sobre este tema não são recentes. Estudos pioneiros como os de Papert e Solomon (1975), entre outros, têm pavimentado o caminho desde os anos 70. Em 2006, Jeannette Wing lançou novos olhares sobre o PC e então "a programação volta à escola" (Kafai e Burke 2013). Em 2014, a própria Wing propôs uma nova definição afirmando que o PC corresponde a processos de pensamento envolvidos na formulação de um problema, que levam à expressão da solução de forma que uma máquina ou uma pessoa possa realizar (Wing, 2014). Neste trabalho, compartilhamos desse entendimento.

Mais do que apenas adotar o PC como atividade/projeto extracurricular ou como parte de uma iniciativa separada, como clubes, a popularização do tema tem sido materializada a partir da sua incorporação aos currículos de tecnologia e de referência, ora de maneira transversal, ora como uma disciplina. Entretanto, existem questões em aberto sobre como desenvolver o PC com crianças. Em 2008, Wing trouxe algumas:

1. Quais são as maneiras efetivas de aprendizagem (ensino) do pensamento computacional pelas (para as) crianças?

2. Quais são os conceitos elementares do pensamento computacional?

3. Como sequenciar adequadamente o ensino dos conceitos para acompanhar a evolução da capacidade de aprendizagem das crianças ao longo dos anos?

4. Qual a melhor forma de integrar ferramentas ao ensino dos conceitos?

Instituições como a Computer Science Teachers Association (CSTA) - ACM ${ }^{1}$, Computing at School ${ }^{2}$, Google ${ }^{3}$, Microsoft ${ }^{4}$, entre outras, têm endereçado as questões (2) e (3), propondo currículos de referência abrangentes, kits de ferramentas, materiais e formações para professores e gestores de escolas públicas e privadas. No entanto, as questões (1) e (4) são mais complexas e a elaboração de respostas baseadas em evidências necessita de um conjunto de esforços em pesquisa, sobretudo empíricos.

No Brasil, os primeiros passos para a consolidação do desenvolvimento do PC se deram em torno das orientações delineadas pela Sociedade Brasileira de Computação (SBC), que propôs o documento Referenciais de Formação em Computação: Educação Básica $^{5}$, visando orientar a incorporação da Ciência da Computação de maneira intracurricular, assemelhando-se a propostas de currículos de referência de outros países

\footnotetext{
${ }^{1}$ https://k12cs.org/

${ }^{2}$ https://www.computingatschool.org.uk/

${ }^{3}$ https://edu.google.com/resources/programs/exploring-computational-thinking/

${ }^{4} \mathrm{https} / / /$ education.microsoft.com/pt-br/course/a41b9507/0

${ }^{5}$ https://www.sbc.org.br/noticias/10-slideshow-noticias/1996-referenciais-de-formacao-em-computacao-educacao-basi
}

V. $19 \mathrm{~N}^{\mathrm{o}}$ 2, Dezembro, 2021

DOI: https://doi.org/10.22456/1679-1916.121361 RENOTE 
${ }^{6}$. Em linhas gerais, o documento da SBC aborda competências e habilidades relacionadas à Ciência da Computação e estabelece um ponto de partida para sua incorporação na educação básica, abrangendo da Educação Infantil ao Ensino Médio, a partir de três eixos: Pensamento Computacional, Mundo Digital e Cultura Digital.

Em um olhar alinhado às habilidades da Base Nacional Comum Curricular (BNCC), o Centro de Inovação para Educação Brasileira (CIEB) apresentou, em 2018, o Currículo de Referência em Tecnologia e Computação para Educação Básica ${ }^{7}$. Similarmente ao documento proposto pela SBC, este currículo trabalha a partir de três eixos (Cultura Digital, Tecnologia Digital e Pensamento Computacional) e descreve habilidades e competências para incorporar a Ciência da Computação na Educação Básica. No mesmo ano, o PC surgiu na BNCC, mas foi apresentado de maneira menos densa, com uma significação pouco esclarecida e predominantemente associado a conceitos matemáticos. Em complemento à BNCC, as Normas sobre Computação na Educação Básica oferecem um olhar mais específico sobre a adoção dos conteúdos de Ciência da Computação ${ }^{8}$.

Neste trabalho, estamos especialmente interessadas em compreender o corpo de conhecimento elaborado sobre o desenvolvimento do PC na Educação Infantil e nos anos iniciais do Ensino Fundamental ao longo dos últimos anos no país, a partir de um MSL. Este trabalho está organizado da seguinte maneira: na Seção 2 são apresentados os trabalhos relacionados e como este trabalho se diferencia dos demais estudos secundários existentes. O processo metodológico deste MSL é descrito na Seção 3. Os resultados obtidos são discutidos na Seção 4. Na Seção 5, são apresentadas as limitações e restrições deste estudo. Por fim, as considerações finais e trabalhos futuros são apresentados na Seção 6.

\section{TRABALHOS RELACIONADOS}

No Brasil, diversas iniciativas envolvendo o ensino de Ciência da Computação e, em especial, abrangendo o PC, têm sido realizadas ao longo dos últimos anos. Em busca de mapear e categorizar estes trabalhos, alguns estudos secundários foram realizados e podem ser classificados em três categorias:

1. abordagens para desenvolvimento do PC (Silva, Pereira e Odakura, 2018; Rodrigues, Aranha e Silva, 2018; Zabot, Andrade e Matos, 2018; Souza e Nunes, 2019; Jesus, Silveira e Palanch, 2019; Avila, Cavalheiro, Bordini e Marques, 2017; Machado e Junior 2019);

2. avaliação do PC (Araujo, Andrade e Guerrero, 2016; Avila et al. 2017) e;

3. perspectivas interdisciplinares para o PC (Nascimento, Santos e Tanzi, 2018; Souza et al. 2019), como Matemática (Barcelos et al. 2015).

Os estudos do primeiro grupo buscam mapear e classificar as diferentes abordagens adotadas para desenvolver o PC, incluindo práticas de programação, por exemplo. Os estudos do segundo grupo mapearam as iniciativas propostas nacionalmente para a avaliação do PC. Por fim, o terceiro grupo apresenta mapeamentos de iniciativas interdisciplinares de desenvolvimento do PC.

\footnotetext{
${ }^{6} \mathrm{https}: / /$ australiancurriculum.edu.au/f-10-curriculum/technologies/introduction/

${ }^{7}$ https://curriculo.cieb.net.br/

${ }^{8}$ http://portal.mec.gov.br/index.php?option=com_docman\&view=download\&alias=182481-texto-referencia-normas-s obre-computacao-na-educacao-basica\&category_slug=abril-2021-pdf\&Itemid=30192
} 
Em linhas gerais, os outros estudos promovem esses mapeamentos considerando a Educação Básica de maneira mais abrangente. O principal diferencial deste trabalho consiste em se concentrar em uma faixa etária mais estreita, em busca de resultados empíricos que nos permitam caracterizar o desenvolvimento do PC no Brasil para este público. Neste trabalho, o escopo foi delimitado apenas para Educação Infantil e anos iniciais do Ensino Fundamental $\left(1^{\circ}\right.$ ao $5^{\circ}$ ano $)$ porque as suas necessidades são específicas com relação aos demais, exigindo abordagens, ferramentas e suportes diferenciados, sobretudo se considerarmos as crianças que ainda estejam em fase de letramento e alfabetização.

\section{TRAJETÓRIAS DE PESQUISA E METODOLOGIA}

Este mapeamento foi realizado a partir das diretrizes trazidas por Kitchenham (2007), com o objetivo responder a seguinte pergunta: Qual o panorama da pesquisa sobre o desenvolvimento do pensamento computacional para crianças pequenas no Brasil?

Para nos auxiliar a responder esta questão principal, definimos as questões auxiliares a seguir, que nortearam a extração e análise dos dados:

- QP1. Quais os tipos de artefatos utilizados?

- QP2. Quais são os tipos de abordagens de ensino adotadas?

- QP3. Quais são os conteúdos, habilidades e competências abordados?

- QP4. Quais são os principais desafios encontrados?

- QP5. Quais são os resultados obtidos?

Estas questões de pesquisa são importantes porque nos ajudam a endereçar as questões trazidas por Wing (2008) sobre quais são as maneiras efetivas de aprendizagem (ensino) do PC pelas (para as) crianças e sobre qual a melhor maneira de integrar as ferramentas ao ensino dos conceitos. Por este motivo, estabelecemos este trabalho de Wing como referencial e a busca foi realizada considerando os anos de 2008 a 2021. Para composição da string de busca, inicialmente propusemos os termos indicados no Quadro 01.

Quadro 01. Termos da String de Busca

\begin{tabular}{|c|c|l|}
\hline id & termo principal & \multicolumn{1}{c|}{ termos alternativos } \\
\hline 01 & programação & $\begin{array}{l}\text { algoritmos I pensamento computacional I raciocínio } \\
\text { computacional I raciocínio lógico I lógica de programação }\end{array}$ \\
\hline 02 & ensino & aprendizado I aprendizagem I desenvolvimento \\
\hline 03 & crianças & Educação Infantil I Ensino Fundamental I Educação Básica \\
\hline
\end{tabular}

No entanto, os testes realizados com a string de busca resultante demonstraram a necessidade de aprimoramento pelos seguintes motivos:

1. Muitos estudos não descrevem apropriadamente quais os conteúdos abordados e embora apresentem uma experiência de ensino de programação, trazem uma variabilidade nos termos adotados, como: raciocínio lógico, raciocínio computacional, algoritmos, entre outros.

2. No Brasil, muitos trabalhos descrevem estudos realizados em ambientes formais de aprendizagem sem distinguir o nível escolar, apresentando apenas as idades 
das crianças participantes e/ou o termo Educação Básica, de maneira muito abrangente. $\mathrm{O}$ mesmo acontece para ambientes informais de aprendizagem.

Por estas razões, criamos uma string de busca simplificada omitindo os termos que referenciam crianças ou nível escolar, tornando a busca mais abrangente:

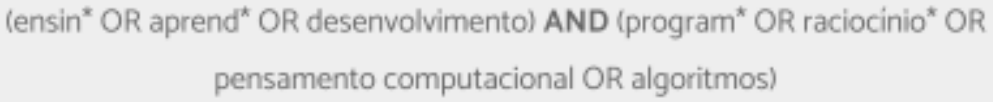

Para realizar a busca, consideramos os principais periódicos e eventos científicos que abordam o tema no Brasil: Revista Novas Tecnologias na Educação (RENOTE), Revista Brasileira de Informática na Educação (RBIE), Simpósio Brasileiro de Informática na Educação (SBIE), Simpósio Brasileiro de Educação em Computação (EDUCOMP), Workshop de Educação em Computação (WEI) ${ }^{9}$, Workshop de Informática na Escola (WIE) e Workshop sobre Ensino em Pensamento Computacional, Algoritmos e Programação (WAlgProg). Os critérios de inclusão e exclusão são apresentados no Quadro 02.

\section{Quadro 02. Critérios de Inclusão e Exclusão}

\begin{tabular}{|c|c|}
\hline Critérios de Inclusão & Critérios de Exclusão \\
\hline $\begin{array}{l}\text { I1. Estudos primários que abordem o ensino de } \\
\text { programação para crianças. }\end{array}$ & E1. Artigos que não foram escritos em português \\
\hline \multirow[t]{8}{*}{$\begin{array}{l}\text { I2. Estudos primários que abordem o } \\
\text { desenvolvimento do pensamento computacional para } \\
\text { crianças. }\end{array}$} & $\begin{array}{l}\text { E2. Artigos convidados, resumidos, tutoriais, palestras, relatórios } \\
\text { de workshop, relatórios técnicos, livros, teses e dissertações. }\end{array}$ \\
\hline & E3. Estudos duplicados/ redundantes de mesma autoria. \\
\hline & E4. Artigos que não foram publicados entre 2008 e 2021. \\
\hline & $\begin{array}{l}\text { E5. Artigos não acessíveis de forma gratuita ou em parceria com } \\
\text { instituições de ensino. }\end{array}$ \\
\hline & $\begin{array}{l}\text { E6. Artigos que não expressem claramente o público-alvo do } \\
\text { estudo desenvolvido. }\end{array}$ \\
\hline & $\begin{array}{l}\text { E7. Artigos que não tenham sido desenvolvidos para crianças } \\
\text { contempladas na faixa etária esperada para a Educação Infantil } \\
\text { e/ou Ensino Fundamental Anos Iniciais (entre } 4 \text { e } 8 \text { anos). }\end{array}$ \\
\hline & E8. Artigos secundários ou terciários. \\
\hline & $\begin{array}{l}\text { E9. Artigos cujo tema central não seja o ensino de programação } \\
\text { e/ou desenvolvimento do pensamento computacional. }\end{array}$ \\
\hline
\end{tabular}

Os trabalhos foram obtidos a partir de uma busca automática nos anais destes periódicos e conferências, exceto pelo WAlgProg. Para este workshop, a busca foi realizada manualmente, a partir do download de todos os artigos publicados desde 2015. Os artigos resultantes de ambas as buscas passaram por uma etapa de pré-seleção, a partir da leitura dos títulos e resumos, sendo analisados quanto aos critérios de inclusão. Posteriormente, os artigos restantes foram analisados quanto aos critérios de exclusão.

${ }^{9}$ Para este workshop os anais estavam disponíveis apenas a partir de 2014.

V. $19 \mathrm{~N}^{\mathrm{o}}$ 2, Dezembro, 2021

DOI: https://doi.org/10.22456/1679-1916.121361 
Por fim, os artigos resultantes destas etapas iniciais, provenientes da busca automática e manual, foram analisados a partir de um conjunto de critérios de qualidade:

- C1. O artigo apresenta os objetivos do estudo?

- C2. O artigo descreve a metodologia proposta?

- C3. O artigo descreve o contexto em que a pesquisa foi realizada?

- C4. O artigo apresenta e discute os trabalhos relacionados?

- C5. Os resultados apresentados são baseados em estudos empíricos?

- C6. O artigo discute os resultados apresentados?

Estes critérios buscavam garantir a qualidade dos artigos selecionados, sendo pontuados conforme a seguinte escala: (0) Não atende, (0.5) Atende parcialmente, (1) Atende. Foram considerados apenas os artigos que pontuaram 5 ou mais.

\subsection{Extração e Síntese dos Dados}

No intuito de responder às questões formuladas, foram extraídos os seguintes dados: metadados, abrangendo ano de publicação, fonte, autores, afiliação, título e resumo; e dados para responder as respectivas questões de pesquisa, como artefatos utilizados, abordagem adotada, conteúdo, habilidades e competências abordados, desafios ou limitações encontrados, e resultados obtidos. Os dados oriundos desta extração estão disponíveis para acesso livremente ${ }^{10}$.

A busca resultou em um total de 977 artigos completos, dos quais 12 foram selecionados para a extração dos dados. A Tabela 01 apresenta os resultados oriundos dos processos de pré-seleção, inclusão e exclusão dos trabalhos mediante análise segundo os critérios de qualidade.

Tabela 01. Quantidade de artigos por etapa do MSL

\begin{tabular}{|l|c|c|c|c|c|c|c|c|}
\hline Etapas/Fonte & SBIE & EDUCOMP & WEI & WIE & WAIgProg & RBIE & RENOTE & Total \\
\hline Pré-seleção & 295 & 26 & 156 & 263 & 159 & 23 & 55 & 977 \\
\hline $\begin{array}{l}\text { Potenciais } \\
\text { candidatos }\end{array}$ & 11 & 02 & 04 & 16 & 16 & 02 & 03 & 53 \\
\hline $\begin{array}{l}\text { Pós-análise } \\
\text { de qualidade }\end{array}$ & 01 & 02 & 04 & 02 & 02 & 01 & 0 & 12 \\
\hline
\end{tabular}

\section{RESULTADOS}

Os resultados obtidos a partir da análise dos artigos são apresentados a seguir, endereçando cada uma das perguntas de pesquisa.

\subsection{Tipos de artefatos utilizados e abordagens de ensino adotadas}

Em linhas gerais, as abordagens de ensino adotadas se concentram no uso de práticas vinculadas ao conceito de Computação Desplugada (Almeida e Costa Junior, 2020; Werlich et al. 2018; Pinheiro et al. 2019) ou ainda em combinações entre estas práticas e programação em plataformas online baseadas em blocos, ou jogos online 
baseados em blocos de programação visual (Silva et al. 2019; Berto, Zaina e Sakata, 2019; França e Tedesco, 2021; Rosa et al. 2021; Godinho et al. 2017). Uma particularidade do trabalho de França \& Tedesco (2021) é a adoção de um livro-jogo, articulando práticas de Computação Desplugada e plataformas de programação em blocos. Zumpichiatti et al. (2021), por sua vez, apresentam uma experiência remota de ensino baseada na articulação entre plataformas online de programação em blocos, vídeos e jogos online, destinada a endereçar os desafios do contexto da COVID-19.

Os trabalhos de Raabe et al. (2018) e Gomes, Melo e Tedesco (2016) adotaram respectivamente brinquedos de programar e apenas jogos digitais online. Trata-se dos dois únicos trabalhos que abordam o desenvolvimento do PC como parte de uma disciplina (regular e extracurricular, respectivamente). Os demais trabalhos são frutos de experiências oriundas de projetos ou programas de extensão ou do Programa Institucional de Bolsas de Iniciação à Docência (PIBID).

\subsection{Conteúdos, habilidades e competências abordadas}

Os estudos analisados não trabalham endereçando especificamente habilidades e competências. Em linhas gerais, os trabalhos endereçam conteúdos básicos de algoritmos e lógica de programação. O trabalho de França e Tedesco (2021), em especial, se debruça sobre os pilares do pensamento computacional: decomposição, generalização, abstração, pensamento algorítmico e avaliação. Similarmente, Rosa et al. (2021) abordam os seguintes pilares do pensamento computacional: decomposição, abstração, algoritmos e reconhecimento de padrões.

\subsection{Desafios encontrados e resultados obtidos}

Em quase sua totalidade, excetuando-se dois trabalhos, os estudos analisados não trouxeram apontamentos sobre desafios encontrados que pudessem nortear futuras pesquisas e os resultados obtidos apontam que as metodologias e artefatos empregados foram adequados e suficientes.

Gomes, Melo e Tedesco (2016) apontam para as dificuldades relacionadas aos elementos de interação da interface dos jogos que indicavam que eles não tinham sido adequadamente projetados para o público a que se destinavam, comprometendo a experiência de uso e de aprendizagem de conceitos como repetição. Elas ressaltam ainda a relevância das narrativas para o público infantil, assim como a necessidade de materiais didáticos projetados para este público. Werlich et al. (2019), por sua vez, afirmaram ter obtido resultados satisfatórios, mas encontraram como desafios a adequação entre as atividades e as faixas etárias, que comprometeram a performance dos alunos em propostas em equipe e individuais.

Em uma perspectiva mais geral, os trabalhos não apresentam considerações e/ou análises sobre desafios encontrados ao longo do percurso da experiência e os resultados relatados asseveram que as práticas foram exitosas.

\section{LIMITAÇÕES E RESTRIÇÕES}

Existem algumas limitações neste estudo que podem ter comprometido os resultados obtidos. Uma destas limitações é que todas as etapas do processo foram conduzidas e executadas por apenas uma pessoa, contrariando as sugestões trazidas por Kitchenham (2007). Estudos importantes podem não ter sido contemplados e vieses pessoais podem ter comprometido desde a pré-seleção até a análise de qualidade. Outro ponto a ser considerado é que muitos artigos apresentavam títulos e resumos que não 
permitiam identificar os objetivos, escopo do trabalho, resultados e/ou contribuições do estudo, dificultando a execução da etapa de pré-seleção.

Em especial, durante o processo de análise de qualidade, notamos que alguns estudos apresentavam conclusões que claramente contradiziam os resultados apresentados, e por este motivo não foram incluídos. Este aspecto nos leva a refletirmos - enquanto comunidade - sobre a maneira como expressamos os resultados obtidos, tentando descrever sempre resultados que possam ser considerados positivos.

\section{CONSIDERAÇÕES FINAIS E DIRECIONAMENTOS FUTUROS}

O desenvolvimento do PC na educação básica tem se tornado realidade em diversos países, com as oportunidades de desenvolver habilidades relacionadas aos conceitos de Ciência da Computação despontando cada vez mais cedo. A quantidade limitada de artigos encontrada no contexto nacional pode significar que temos um vislumbre pouco fidedigno do cenário de desenvolvimento do PC para crianças, embora entendamos que existem iniciativas de longa duração como as promovidas pelo Instituto Ayrton Senna ${ }^{11}$ e que não foram desenvolvidas no meio acadêmico. Além disso, estes resultados sugerem que podemos estar desconsiderando importantes desafios enquanto oportunidades de pesquisa neste tema com esta faixa etária.

Se considerarmos o espaço temporal de 14 anos desde a proposição das questões sobre como desenvolver o PC com crianças por Wing (2008), percebemos que ainda temos um longo caminho a percorrer, especialmente quando levamos em consideração que outros países já possuem currículos que abordam a faixa etária da Educação Infantil e anos iniciais do Ensino Fundamental. No Brasil, a BNCC ainda contempla o PC de maneira superficial, e uma das principais referências é o currículo proposto pelo CIEB.

Um outro ponto de atenção é a necessidade de propor atividades que sejam condizentes com a faixa etária considerando aspectos do desenvolvimento cognitivo, motor e espacial. Werlich et al. (2018), por exemplo, descrevem que os alunos do $1^{\circ}$ ano EF sentiram dificuldades em lidar com os conceitos de direita e esquerda. No entanto, crianças nesta faixa etária ainda estão desenvolvendo noções de lateralidade e a atividade proposta precisava de adaptação para introduzir o conteúdo pela primeira vez. Situações como esta servem como alerta para uma outra necessidade latente, corroborada por Gomes, Melo e Tedesco (2016), Silva et al. (2019) e Zumpichiatti et al. (2021): a produção de materiais didáticos, propostas de atividades e recursos educacionais para apoiar o desenvolvimento do PC com crianças pequenas.

Para ampliar nossa visão, em trabalhos futuros buscaremos proporcionar um olhar mais integrativo e interdisciplinar. Esperamos ampliar este MSL incluindo outras conferências e periódicos nacionais que versem sobre este mesmo tema em outras áreas, como Educação. Complementarmente, esperamos expandir este olhar para o cenário internacional para que possamos compreender e comparar os avanços reportados no Brasil e em outros países, ampliando as discussões entre os contextos mundial e nacional do ensino de programação para iniciantes com ênfase na Educação Infantil e no Ensino Fundamental (Ramos et al. 2015).

11

https://institutoayrtonsenna.org.br/content/dam/institutoayrtonsenna/radar/estante-educador/instituto-ayrton-senna-ed ucacao-integral-por-meio-do-pensamento-computacional.pdf

V. $19 \mathrm{~N}^{\circ}$ 2, Dezembro, 2021 RENOTE

DOI: https://doi.org/10.22456/1679-1916.121361 


\section{REFERÊNCIAS}

Araujo, A., Andrade, W., \& Guerrero, D. (2016). Um Mapeamento Sistemático sobre a Avaliação do Pensamento Computacional no Brasil. Anais dos Workshops do Congresso Brasileiro de Informática na Educação, 5(1), 1147. doi:http://dx.doi.org/10.5753/cbie.wcbie.2016.1147

Avila, C., Cavalheiro, S., Bordini, A., \& Marques, M. (2017). O Pensamento Computacional por meio da Robótica no Ensino Básico - Uma Revisão Sistemática. Brazilian Symposium on Computers in Education (Simpósio Brasileiro de Informática na Educação - SBIE), 28(1), 82. doi:http://dx.doi.org/10.5753/cbie.sbie.2017.82

Avila, C., Cavalheiro, S., Bordini, A., Marques, M., Cardoso, M., \& Feijo, G. (2017). Metodologias de Avaliação do Pensamento Computacional: uma revisão sistemática. Brazilian Symposium on Computers in Education (Simpósio Brasileiro de Informática na Educação - SBIE), 28(1), 113. doi:http://dx.doi.org/10.5753/cbie.sbie.2017.113

Barba, L. 2016. Computational Thinking: I do not think it means what you think means. Disponível em: http://bit.ly/2EASvMj. Acessado em: 30/10/2021

Barcelos, T., Muñoz, R., Acevedo, R., \& Silveira, I. (2015). Relações entre o Pensamento Computacional e a Matemática: uma Revisão Sistemática da Literatura. Anais dos Workshops do Congresso Brasileiro de Informática na Educação, 4(1), 1369. doi:http://dx.doi.org/10.5753/cbie.wcbie.2015.1369

Blikstein, P. 2008. O pensamento computacional e a reinvenção do computador na educação. Disponível em: http://bit.ly/2H7LgKi. Acessado em: 30/10/2021.

Bordini, A., Avila, C., Marques, M., Foss, L., \& Cavalheiro, S. (2017). Pensamento Computacional nos Ensinos Fundamental e Médio: uma revisão sistemática. Brazilian Symposium on Computers in Education (Simpósio Brasileiro de Informática na Educação - SBIE), 28(1), 123. doi:http://dx.doi.org/10.5753/cbie.sbie.2017.123

Carvalho, J., Netto, J., \& Almeida, T. (2017). Revisão Sistemática de Literatura sobre Pensamento Computacional por Meio de Objetos de Aprendizagem. Brazilian Symposium on Computers in Education (Simpósio Brasileiro de Informática na Educação - SBIE), 28(1), 223. doi:http://dx.doi.org/10.5753/cbie.sbie.2017.223

Silva, K., Pereira, N.P. and Odakura, V., 2018. Mapeamento Sistemático: estratégias para o ensino-aprendizagem do Pensamento Computacional no Brasil. In XXIII Congreso Internacional de Informática Educativa (pp. 319-329).

Guzdial, M. (2015). Learner-centered design of computing education: Research on computing for everyone. Synthesis Lectures on Human-Centered Informatics, 8(6), $1-165$.

IFTF - Institute for the Future. Everyone is a programmer: Making the World a Control System. When everything is programmable: Life in a Computational Age. 2009.

Jesus, A., Silveira, I., \& Palanch, W. (2019). Desenvolvimento do Pensamento Computacional por Meio da Colaboração: uma revisão sistemática da literatura. Revista 
Brasileira de Informática na Educação, 27(02), 69.

doi:http://dx.doi.org/10.5753/rbie.2019.27.02.69

Kafai, Y. B., \& Burke, Q. (2013). Computer programming goes back to school. Phi Delta Kappan, 95(1), 61-65.

Kitchenham, B. A. (2007) Guidelines for performing Systematic Literature Reviews in Software Engineering.

Machado, J., \& Junior, A. (2019). Utilização de jogos como ferramenta para auxiliar o desenvolvimento do Pensamento Computacional: uma revisão sistemática. Anais do Workshop de Informática na Escola, 25(1), 217-226.

doi:http://dx.doi.org/10.5753/cbie.wie.2019.217

Nascimento, C., Santos, D., \& Tanzi, A. (2018). Pensamento Computacional e Interdisciplinaridade na Educação Básica: um Mapeamento Sistemático. Anais dos Workshops do Congresso Brasileiro de Informática na Educação, 7(1), 709. doi:http://dx.doi.org/10.5753/cbie.wcbie.2018.709

Ortiz, J., \& Pereira, R. (2018). Um Mapeamento Sistemático Sobre as Iniciativas para Promover o Pensamento Computacional. Brazilian Symposium on Computers in Education (Simpósio Brasileiro de Informática na Educação - SBIE), 29(1), 1093. doi:http://dx.doi.org/10.5753/cbie.sbie.2018.1093

Ramos, V., Wazlawick, R., Galimberti, M., Freitas, M., \& Mariani, A. C. (2015, October). A Comparação da Realidade Mundial do Ensino de Programação para Iniciantes com a Realidade Nacional: Revisão sistemática da literatura em eventos brasileiros. In Brazilian Symposium on Computers in Education (Simpósio Brasileiro de Informática na Educação-SBIE) (Vol. 26, No. 1, p. 318).

Rodrigues, S., Aranha, E., \& Silva, T. (2018). Computação Desplugada no Ensino de Programação: Uma Revisão Sistemática da Literatura. Brazilian Symposium on Computers in Education (Simpósio Brasileiro de Informática na Educação - SBIE), 29(1), 417. doi:http://dx.doi.org/10.5753/cbie.sbie.2018.417

Sinésio Ferris da Silva, I., \& Pontual Falcão, T. (2021). UMA PESQUISA DOCUMENTAL SOBRE O PENSAMENTO COMPUTACIONAL NO ENSINO SUPERIOR: ANÁLISE DOS PROJETOS PEDAGÓGICOS DOS CURSOS DE LICENCIATURA EM COMPUTAÇÃO NO BRASIL: A DOCUMENTAL RESEARCH ABOUT COMPUTATIONAL THINKING IN HIGHER EDUCATION: ANALYSIS OF PEDAGOGICAL PROJECTS OF COMPUTER SCIENCE TEACHER EDUCATION PROGRAMS IN BRAZIL. Revista Contexto \& Educação, 36(114), 54-71.https://doi.org/10.21527/2179-1309.2021.114.54-71

Souto, M., \& Tedesco, P. (2017). Uma Revisão sistemática da Literatura sobre conhecimentos, habilidades, atitudes e competências desejáveis para auxiliar a aprendizagem de programação. Anais dos Workshops do Congresso Brasileiro de Informática na Educação, 6(1), 1162.

doi:http://dx.doi.org/10.5753/cbie.wcbie.2017.1162 
Souza, F., \& Nunes, M. (2019). Práticas e resultados obtidos na aplicação do Pensamento Computacional Desplugado no ensino básico: Um Mapeamento Sistemático. Brazilian Symposium on Computers in Education (Simpósio Brasileiro de Informática na Educação - SBIE), 30(1), 289.

doi:http://dx.doi.org/10.5753/cbie.sbie.2019.289

Souza, F., Leite, R., Brito, C., Villela, M., \& Santos, C. (2019). O desenvolvimento do Pensamento Computacional além do ensino em ciências exatas: uma revisão da literatura. Brazilian Symposium on Computers in Education (Simpósio Brasileiro de Informática na Educação - SBIE), 30(1), 528.

doi:http://dx.doi.org/10.5753/cbie.sbie.2019.528

Stephenson, C. et al. The new CSTA K--12 computer science standards. In: Proceedings of the 17th ACM annual conference on Innovation and technology in computer science education. ACM, 2012. p. 363- 364.

Wing, J. M. (2006) Computational thinking. Communications of the ACM, v. 49, n. 3, p. 33-35.

Wing, J. M. (2008). Computational thinking and thinking about computing.

Philosophical Transactions of the Royal Society A: Mathematical, Physical and Engineering Sciences, 366(1881), 3717-3725.

Zabot, D., Andrade, S., \& Matos, E. (2018). Raciocínio Computacional e Jogos Digitais: categorias e mecânicas. Brazilian Symposium on Computers in Education (Simpósio Brasileiro de Informática na Educação - SBIE), 29(1), 765. doi:http://dx.doi.org/10.5753/cbie.sbie.2018.765

Zanetti, H., Borges, M., \& Ricarte, I. (2016). Pensamento Computacional no Ensino de Programação: Uma Revisão Sistemática da Literatura Brasileira. Brazilian Symposium on Computers in Education (Simpósio Brasileiro de Informática na Educação - SBIE), 27(1), 21. doi:http://dx.doi.org/10.5753/cbie.sbie.2016.21

\section{ARTIGOS SELECIONADOS}

Almeida, W., \& Costa Junior, A. (2020). A Aplicação de uma Sequência Didática no Processo de Desenvolvimento do Pensamento Computacional com Alunos do $4^{\circ}$ Ano do Ensino Fundamental I. In Anais do XXVI Workshop de Informática na Escola, (pp. 11-20). Porto Alegre: SBC. doi:10.5753/cbie.wie.2020.11

Berto, L., Zaina, L., \& Sakata, T. (2019). Metodologia Para Ensino do Pensamento Computacional para Crianças Baseada na Alternância de Atividades Plugadas e Desplugadas. Revista Brasileira de Informática na Educação, 27(02), 01. doi:http://dx.doi.org/10.5753/rbie.2019.27.02.01

França, R., \& Tedesco, P. (2021). Corporeidade, ludicidade e contação de história na promoção do pensamento computacional na escola. In Anais do Simpósio Brasileiro de Educação em Computação, (pp. 132-142). Porto Alegre: SBC. doi:10.5753/educomp.2021.14479

Godinho, J., Torres, K., Batista, G., Andrade, E., \& Gomide, J. (2017). Projeto Aprenda a Programar Jogando: Divulgando a Programação de Computadores para Crianças e V. $19 \mathrm{~N}^{\circ} 2$, Dezembro, 2021 DOI: https://doi.org/10.22456/1679-1916.121361 RENOTE 
Jovens. In Anais do XXV Workshop sobre Educação em Computação. Porto Alegre: SBC. doi:10.5753/wei.2017.3553

Gomes, T., Melo, J., \& Tedesco, P. (2016). Jogos Digitais no Ensino de Conceitos de Programação para Crianças. Brazilian Symposium on Computers in Education (Simpósio Brasileiro de Informática na Educação - SBIE), 27(1), 470. doi:http://dx.doi.org/10.5753/cbie.sbie.2016.470

Pinheiro, J., Godinho, J., Guedes , Y., Cardoso, G., Zumpichiatti, D., \& Gomide, J. (2019). Programa(ação): Atividades lúdicas para ensino de programação em escolas públicas. In Anais do XXVII Workshop sobre Educação em Computação, (pp. 91-100). Porto Alegre: SBC. doi:10.5753/wei.2019.6620

Raabe, A., de Jesus, E., da Silva, E., \& Couto, N. (2018). Percepção dos Estudantes sobre a Implantação de uma Disciplina Regular de Pensamento Computacional em um Colégio de Educação Básica. In Anais do XXVI Workshop sobre Educação em Computação. Porto Alegre: SBC. doi:10.5753/wei.2018.3522

Raabe, A., Santana, A., Martins, R., Souza, F., Rosário, T., \& Silva, R. (2017). RoPE Brinquedo de Programar e Plataforma de Aprender. Anais do Workshop de Informática na Escola, 23(1), 1119-1128. doi:http://dx.doi.org/10.5753/cbie.wie.2017.1119

Rosa, Y., Reiser, R., Oliveira, P., Foss, L., Cavalheiro, S., Du Bois, A., Piana, C., \& Mazzini, A. (2021). PC-Câmbio: Proposta de Atividade Lúdica e Desplugada Aplicando a Metodologia do Pensamento Computacional. In Anais do Simpósio Brasileiro de Educação em Computação, (pp. 227-236). Porto Alegre: SBC. doi:10.5753/educomp.2021.14489

Silva, A., Oliveira, C., Ilnicki, K., Madeira, C., \& Campos, A. (2019). Pensamento computacional como auxílio para estimular a noção espacial das crianças do Ensino Fundamental. Anais dos Workshops do Congresso Brasileiro de Informática na Educação, 8(1), 387. doi:http://dx.doi.org/10.5753/cbie.wcbie.2019.387

Werlich, C., Crema, C., Kemczinski, A., \& Gasparini, I. (2018). Pensamento Computacional no Ensino Fundamental I: um estudo de caso utilizando Computação Desplugada. Anais dos Workshops do Congresso Brasileiro de Informática na Educação, 7(1), 719. doi:http://dx.doi.org/10.5753/cbie.wcbie.2018.719

Zumpichiatti, D., SantClair, G., Moreira, J., \& Gomide, J. (2021). Um Relato Sobre a Experiência do Ensino de Programação para Crianças e Jovens de Forma Remota. In Anais do XXIX Workshop sobre Educação em Computação, (pp. 161-170). Porto Alegre: SBC. doi:10.5753/wei.2021.15907 